\title{
Cardiac 31 P MRS analysis development: Improved fitting of 2, 3 DPG
}

\author{
Sairia Dass ${ }^{* *}$, Damian Tyler ${ }^{2}$, Stefan Neubauer ${ }^{1}$, Kieran Clarke², Lowri E Cochlin ${ }^{2}$ \\ From 16th Annual SCMR Scientific Sessions \\ San Francisco, CA, USA. 31 January - 3 February 2013
}

\section{Background}

31P MRS is an important tool for the measurement of in vivo energetics via the ratio $\mathrm{PCr} / \mathrm{ATP}$. Low signal-to-noise (31P molar receptivity is $1 / 14$ that of $1 \mathrm{H}$ ) coupled with low concentration of high-energy phosphates can result in high measurement variability. 31P MRS of human heart often contains signals originating from blood i.e. 2,3DPG and ATP. Measurement of myocardial PCr/ATP thus requires subtraction of blood derived ATP signal. The aim of this work was to improve blood correction by applying prior knowledge (PK) of molecular spectral parameters to the 2,3DPG signal. We demonstrate that accurate spectral fitting of 2,3DPG prevents proliferation of fitting errors through blood correction, resulting in improved accuracy of PCr/ATP measurement.

\section{Methods}

A review of the literature for spectral information about 2,3DPG was employed to guide changes to the existing PK for 2,3DPG, Table 1. Sixteen $31 \mathrm{P}$ spectra were simulated in jMRUI, PCr/ATP ratio fixed at 2.00 with $20 \%$ and $40 \%$ noise added. Forty eight datasets from $8 \mathrm{HCM}$, $8 \mathrm{DCM}$ and 8 normal controls were analyzed. The original and the new 2,3DPG fitting PKs were applied to the each simulated and patient dataset.

\section{Results}

Simulated Data

In the data with $20 \%$ noise, there were no differences in the mean and standard deviations (SD) of the PCr/ATP ratios with the original ( $2.02 \pm 0.13)$ and new 2,3 DPG fit $(2.03 \pm 0.12, \mathrm{P}=0.3)$. However, with $40 \%$ noise, both the mean and SD of the $\mathrm{PCr} / \mathrm{ATP}$ ratios using the original 2,3DPG fit, were significantly higher than with using the new 2,3DPG fit (original fit: PCr/ATP $2.77 \pm 0.99$; new fit:
Table $1 \mathrm{~A}$ summary of the differences between the original and new prior knowledge for 2, 3 DPG.

\begin{tabular}{cccc}
\hline & PARAMETER & OLD PK & NEW PK \\
\hline 2 DPG & Amplitude estimate & estimate & Fixed 3 DPGX0.8 \\
\hline Relative Phase & Flxed 0 & Fixed 0 \\
\hline Line Width & estimate & 20-100 \\
\hline Frequency & Soft constraint & 5.01-6.01 \\
\hline Shape & Lorentizian & Lorentizian \\
\hline PARAMETER & OLD PK & NEW PK \\
\hline 3 DPG & Amplitude estimate & estimate & estimate \\
\hline Relative Phase & Flxed 0 & Flxed 0 \\
\hline Line Width & estimate & 2 DPGx1 \\
\hline Frequency & Soft constraint & 6.02-6.81 \\
\hline Shape & Lorentizian & Lorentizian
\end{tabular}

PCr/ATP 2.33 $\pm 0.46, \mathrm{p}<0.05)$. The mean difference of the measured PCr/ATP ratio and the actual ratio of 2.00 was $0.77 \pm 0.99$ for the original fit and $0.33 \pm 0.46$ for the new fit, $\mathrm{P}=0.02$.

\section{Patient Data}

There was no difference in the mean PCr/ATP with the different PKs (original fit: HCM 1.96 \pm 0.53 , DCM 1.47 \pm 0.28 , normal $2.26 \pm 0.32$; new fit: HCM $1.80 \pm 0.33$, DCM $1.51 \pm 0.20$, normal $2.22 \pm 0.28, P>0.1)$. However, with the new PK, there was a substantial improvement in the coefficient of variance of the 2,3DPG fit in all groups (original fit vs. new fit: $\mathrm{HCM} 24 \%$ vs $15 \% \mathrm{P}<0.005$; DCM $22 \%$ vs $12 \% \mathrm{P}<0.005$; normal controls $23 \%$ vs $15 \% \mathrm{P}<0.0005$, Figure 1). This translated into a reduction in the $\mathrm{SD}$ of the PCr/ATP measurements in all 3 groups. In HCM and DCM, based on this pilot data, in order to detect a $10 \%$ change in PCr/ATP with an intervention ( $\alpha 0.05$, power 


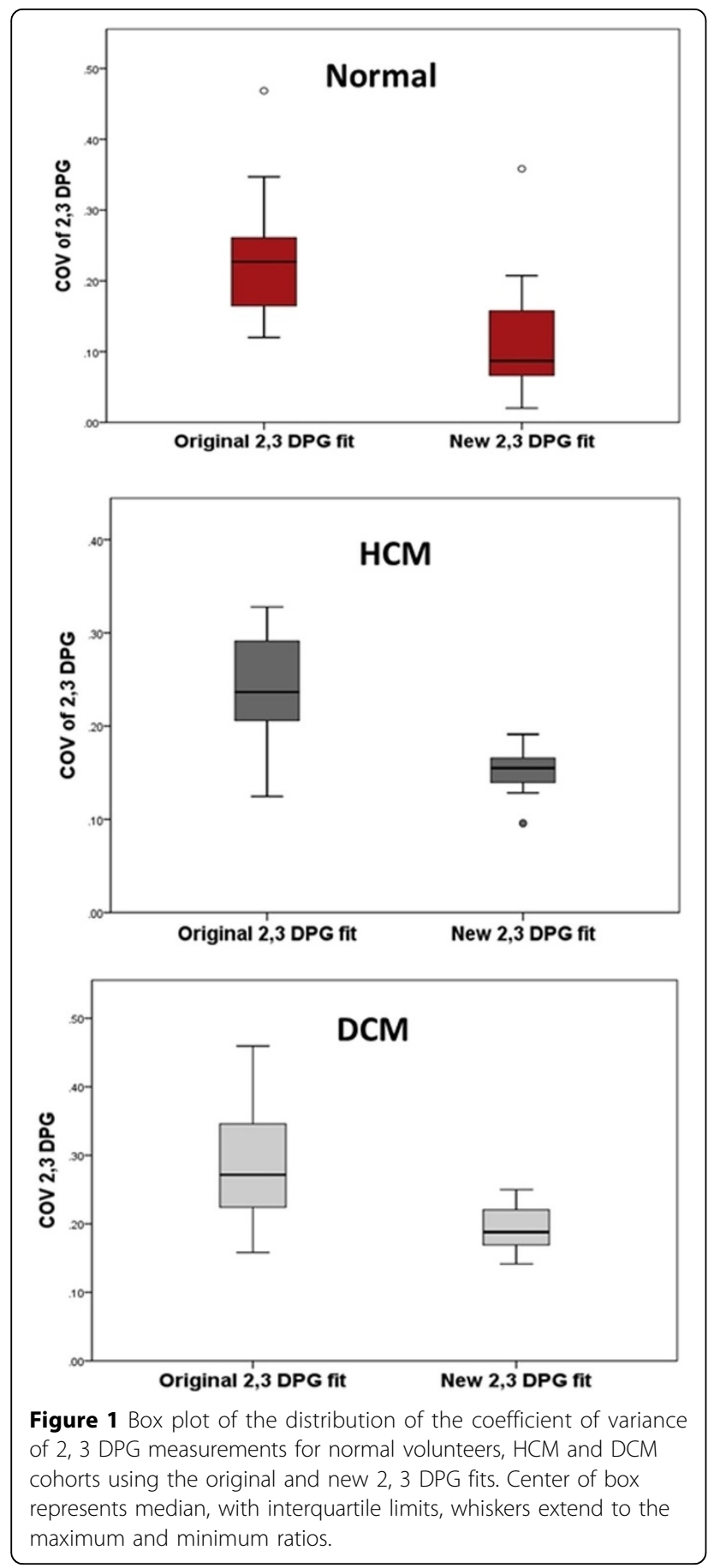

$80 \%)$, the number of subjects needed with the new 2,3DPG PK is reduced from 59 to 27 in HCM and 28 to 14 in DCM.

\section{Conclusions}

31P MRS is a valuable tool for assessment of myocardial metabolism.. This work developed an improved fit for 2,3DPG hence minimising propagation of errors to the final PCr/ATP calculation. The quality of this outcome is reflected in the power calculations which illustrate an almost $50 \%$ reduction of sample size required to statistically demonstrate a change in $\mathrm{PCr} / \mathrm{ATP}$ with an intervention in HCM and DCM.

\section{Funding}

British heart foundation.

\section{Author details}

'OCMR, John Radcliffe Hospital, Oxford, UK. ²Department of Physiology, Anatomy and Genetics, Oxford University, Oxford, UK.

Published: 30 January 2013

doi:10.1186/1532-429X-15-S1-P219

Cite this article as: Dass et al:: Cardiac 31 P MRS analysis development: Improved fitting of 2, 3 DPG. Journal of Cardiovascular Magnetic Resonance 2013 15(Suppl 1):P219.
Submit your next manuscript to BioMed Central and take full advantage of:

- Convenient online submission

- Thorough peer review

- No space constraints or color figure charges

- Immediate publication on acceptance

- Inclusion in PubMed, CAS, Scopus and Google Scholar

- Research which is freely available for redistribution 